\title{
PHENOTYPIC CLASSIFICATION OF PATIENTS TREATED WITH OSTEOARTHRITIS
}

lais roberta garcia bernardini venancio (pontificia universidade catolica de são paulo, Sorocaba, SP, Brasil), martina fernanda marcolino (pontificia universidade catolica de são paulo, Sorocaba, SP, Brasil), josé eduardo martinez (pontificia universidade catolica de são paulo, Sorocaba, SP, Brasil)

\section{BACKGROUND}

\section{ABSTRACT}

Osteoarthritis is an important issue for both the individual and society [1]. Its public health impact continues to grow due to the ageing population, the rising prevalence of obesity and the lack of definitive treatments to prevent or halt the progress of the disease [2]. However, osteoarthritis is difficult to define, and a better understanding of its pathophysiology is required $[1,2]$. What all forms of osteoarthritis and related disorders have in common is a loss of cartilage associated with bone features such as osteophytes and subchondral bone sclerosis [3]. In addition to improving our understanding of the disease, classifying the different clinical and structural phenotypes of osteoarthritis will allow for more direct targeting of treatments, depending on whether the predominate structural changes are in cartilage, bone, or synovial tissue. Nevertheless, there is currently no consensus on the subgrouping of osteoarthritis into these phenotypes and they are not yet fully characterised [4]

\section{MATERIALS AND METHODS}

Method: cross-sectional study. It was analyzed records of 113 patients attended in the last two years in the osteoarthritis clinic of a university outpatient rheumatologic unit. Patients whose records were incomplete were excluded. Each patient was classified according to European Society for Clinical and Economic Aspects of Osteoporosis, Osteoarthritis and Musculoskeletal Diseases (ESCEO).

\section{RESULTS}

Results: It was studied 113 patients, with female predominance (97\%), mean age of $63=/-6,3$ years old, cumulative joint distribution showed: $8 \%$ hands, $12,4 \%$ cervical and lumbar spine, $30 \%$ generalized, $8 \%$ monoarticular and $41,6 \%$ oligoarticular From a phenotype point of view, the patients were distributed as follows: 15,0\% "aging", 4,4\% "post traumatic", 41,6\% "metabolic" , 24,8\% "pain" and "undetermined" $14,1 \%$. The main characteristics of each group will be presented.

\section{CONCLUSION}

Conclusion: There is a predominance of the metabolic phenotype followed by the pain. In a minor part of the patients, it was not possible to classify in one phenotype. 